\title{
La Vie Philosophique en France
}

QUATREMERE DE QUINCY, Antoine-Chrysotôme. Considérations morales sur la destination des ouvrages de l'art. Paris: Librairie Arthème Fayard, 1989, Pp.250.

"Cet opuscule, anciennement composé," nous dit l'auteur dans la préface, "l'a été sans aucun autre but de ma part que d'éprouver la valeur et l'effet de certaines idées, détachées de l'ensemble d'un Traité plus considérable, sur l'effet poétique des ouvrages de l'Art." D'abord lus à la classe des Beaux Arts de l'Institut et écoutés "avec indulgence", ces morceaux deviennent une dizaine d'années plus tard, les Considérations morales sur la destination des ouvrages de l'art, avec comme sous-titre, "ou de l'influence de leur emploi sur le génie et le goût de ceux qui les produisent ou qui les jugent, et sur le sentiment de ceux qui en jouissent et en reçoivent les impressions." L' ouvrage, publié à l'origine en 1815 (chez Crapelet) paraît aux Editions Fayard en 1989 suivi de sept lettres écrites de Londres et adressées à $\mathbf{M}$. Canova et de sept lettres au Général Miranda sur le préjudice qu'occasionnent aux arts et à la science le déplacement des monumens de l'art de l'Italie.

Les Considérations morales sont divisées en deux parties; la première est consacrée, principalement, au développement d'une théorie esthétique, basée sur trois principles essentiels, l'imitation, l'utilité et la morale. "L'Art et l'ouvrage (seront) utiles, d'une utilité morale, quand l'imitation, au lieu de viser uniquement au plaisir des sens, a pour effet spécial d'agrandir la pensée, de réveiller en nous de nobles affections. . ." (p.18). Les beaux ouvrages demandent pour être bien reproduits plus que de simples procédés mécaniques mais "une puissance d'amour que rien ne supplée et qui est ellemême le supplément de beaucoup de facultés" (p.25). L'auteur s'érige avec véhémence contre les amateurs qui ne voient dans les oeuvres d'art qu'une façon de s'enricher--ainsi dégradant l'objet--et contre les artistes qui vendent leur talent car "faire vivre les Arts, c'est rendre leurs ouvrages utiles, ce qui est fort différent de faire vivre les artistes par des ouvrages qui ne le sont pas" (p.27). D'ailleurs la création demande un talent special et les " chefs-d'oeuvre de génie" ne peuvent "ni se commander exprès, ni s'obtenir à volonté" (p.16) et ne devraient pas être confondus avec les "productions inutiles, ou les frivolités de luxe," (p.16). La création, d'après de Quincy, ne semblerait pas être un besoin physiologique qui demande à être satisfait mais plutôt un jeu de l'imagination produit par la connaissance, la jouissance, et "la passion des ouvrages de l'Art,.. qu'il importe de développer et d'exciter chez un peuple, 
pour fortifier chez l'artiste la puissance créatrice, qui réside dans le sentiment" (p.42). Cela ne revient pas à dire, comme on aurait pu le penser, que tout oeuvre d'art doit nécessairement être exposée dans un musée. Bien au contraire, un mélange du médiocre et du pire, ajouté aux collections d'élite, aurait son utilité, "dans l'échelle des points de comparaison qui servent à apprécier le bon" (p.39). Et s'il y a une technique dans l'appréciation du bon et du beau, il y a certes aussi, pour l'auteur, une façon de faire intervenir et de demander cette appréciation. One ne peut se contenter de ne voir dans les musées que "des collections destinées à l'enseignement et la copie" (p.37); pas plus qu'on ne doit insister sur l'exposition seule des grands talents du temps passé dans ces collections d'élite qui semble trop apprendre aux amateurs "quelle distance les sépare "des talens de l'âge où l'on vit" car bientôt "une sorte de superstition pour ce qui est ancien (porterait) à condamner trop rigoureusement ce qui est moderne" (p.40). Il n'est pas question non plus de faire de ces musées des "conservatoires" où sont amassées au grand détriment du public, des fragments décomposés qui ne sont utiles qu'en simples sujets de critiques:

En classer méthodiquement les débris, et faire d'une telle réunion un cours pratique de chronologie moderne; c'est pour une raison existante, se constituer en état de nation morte; c'est de son vivant assister à ses funérailles; c'est tuer l'art pour en faire l'histoire; ce n'est point en faire l'histoire, mais l'épitaphe" p.48).

"D'où dépendent l'impression du plaisir et la sensation du beau" (p. 49) est le sujet de la deuxième partie de cet essai. "Résultat d'une harmonie inappréciable... dont la délicatesse échappe à l'intelligence" (p. 49) cette qualité serait indéfinissable car les causes en restent inaperçues. Laissant libre cours à son imagination, c'est à l'artiste de créer un ensemble d'accompagnements aussi plaisants que, parfois, inattendues et où la nature peut ainsi être enjolivée, même corrompue, au gré de l'interprétation, et l'expression de valeurs esthétiques concrétisé dans la composition de l'espace. Avec beaucoup de soins, l'artiste prépare "tous ces charmes accessoires autour de son idole":

Tantôt il le place sous un bosquet que l'amour a tissu de myrtes et de roses. Tantôt il lui bâtit un temple qu'un bois sacré précède: le portes entr'ouvertes excitent le désir d'y pénétrer. Au dedans brille l'or, l'ivoire et la pourpre. Un voile mystérieux cache sa divinité. Au son des accords sacré tombe le voile, l'encens fume, et au milieu des vapeurs d'un nuage religieux 
brille tout-à-coup la déesse" (p.51).

Quatremère de Quincy ne nomme pas d'artistes contemporains motivé par cette technique mais Dominique Ingres qui, lui aussi, enseigna à l'ecole des Beaux-Arts (en 1834) ne manque pas, tout spécialement dans l' Odalisque et l'esclave, d'une sensualité raffinée, de créer d"efficaces illusions." Ce même tableau n'avait-il pas été dans sa première version peint avec un mur dans l'arrière plan plutôt que donnant sur un jardin!

Inclus dans ce recueil sont deux séries de sept lettres chacune, les premières adressées à $\mathbf{M}$. Canova, sur les marbres d'Elgin et les secondes au Général Miranda, "sur le préjudice qu'occasionnens aux arts et à la science le dépla- cement des monumens de l'art de l'Italie, le démembrement de ses écoles et la spoliation de ses collections, galeries, musées, etc ".

Adoptant la devise, diviser c'est détruire, De Quincy--qui avait publié en 1803 une longue dissertation sur L'Architecture égyptienne considérée dans son origine, ses principes et son goût et comparée sous les mêmes rapports à larchitecture grecque (Paris: Barrois, In-4o, xii-268 p.)--discute, en 1796, "le tort irréparable que causerait à la science et à l'art une imprudente convoitise des trésors de l'antiquité que renferme l'Italie, et surtout Rome" (p.205). Enlever tous ces trésors de leur lieu de naissance est leur enlever une partie de leur valeur et l'Italie étant elle-même un grand musée, on ne pourra suppléer au voyage à Rome; hence, De Quincy se montre contre l'enlèvement des anciens trésors de l'Italie, et les "grands emmagasinemens" dans les pays étrangers sur "la présumtion de (la) savantise" de quelques hommes "plus dangereux par leurs demi-connaissances, que les ignorans mêmes" (p.210).

Faisant à première vue complètement demi-tour dans ses Lettres écrites de Londres à Canova, Quatremère défend le transport de certaines sculptures du Temple de Minerve d'Athènes à Londres, au British Muséum. Il explique que, du point de vue de la morale, des circonstances politiques et principalement pour la conservation même de ces ouvrages leur enlèvement était nécessaire. Situées trop loin pour être vues et appréciées du public, les sculptures enlevées des ruines du Parthénon, étaient menacées de disparaitre complètement. Mieux valait les transporter à Londres, les remettre en état afin de les conserver et les présenter au public à leur plus grand avantage. Faisant preuve d'une compréhension moderne de la critique, l'auteur dit que "l'ouvrage doit être jugé selon l'esprit dans lequel il a été fait; il y a, pour chaque genre de travail, un esprit de critique qui lui est assorti." Et ajoute-t-il, dans le fond, tout ouvrage porte avec soi la propriété d'instruire le spectateur 
de la manière dont il doit être vu et jugé; et nul ouvrage plus que (la frise du Parthénon) n'est doué de cette propriété" (p. 117). Suit une description très sophistiquée des 180 pieds que forme cette composition (probablement de plus de 450 pieds à l'origine). Expliquée également est la méthode utilisée pour détacher avec facilité, et sans l'endommager, de la coulisse qui l'avait reçu, chaque groupe des métopes.

L'opinion exprimée par Quatremère de Quincy dans les Considérations morales sur la destination des ouvrages de l'art est sans équivoque; l'oeuvre d'art doit être conservée dans l'ambiance qui lui est propre afin qu'elle ne perde pas "une portion considérable de (sa) beauté relative" (p.53). Mais de là il y a un grand pas, qui ne doit pas être franchi, à les laisser déprécier ou les ignorer, loin ou delà de la vue publique. Autant les démanteler et les reconstruire avec tous les soins et connaissances nécessaires pour la jouissance et compréhension des artistes et connaisseurs car, insiste, Quatremère de Quincy:

en vain dira-t-on que les beaux ouvrages n'ont besoin pour plaire que de leur propre mérite, je répondrai oui pour les savans, non, pour le reste des hommes. Je répondrai encore oui pour les chefs-d'oeuvre, non, pour le grand nombre des ouvrages (p. 79).

Les Considérations morales sur la destination des ouvrages de l'art est en fait un traité sur la science du beau dans la nature et dans l'art mais nulle part dans l'ouvrage n'est mentionné le mot "esthétique". Peut-être ce mot produisait-il sur l'auteur, comme sur Valéry, (dans Variété IV), "un effet d'éblouissement, si ce n'est d'intimidation."

\section{Annonces et nouvelles}

Nous sommes heureux de publier ci-joint une bibliographie d'oeuvres philosophiques publiées de 1984 à 1986. Elle sera suivie dans notre prochain numéro par une bibliographie complémentaire se rapportant aux années 1986 à 1990 et également dressée grâce aux bons soins du Professeur Racevslis, de Wright State University. Nous le remercions chaleureusement pour ce travail. 